\title{
Increased minimum alveolar concentration- awake of Sevoflurane in women of breast surgery with sleep disorders
}

\author{
Yuanyuan Cao ${ }^{1,2}$, Lei Zhang ${ }^{2}$, Xiaohui Peng ${ }^{2}$, Yun Wu', Qunlin Zhang ${ }^{3}$, Erwei Gu² and Ye Zhang ${ }^{1 *}$ (D)
}

\begin{abstract}
Background: Sleep disorders are commonly encountered in clinic. Evidences showed that sleep deprivation may modulate the effectiveness of general anesthetics in rats. However, this phenomenon has not been explored in humans. The study aimed to investigate whether the hypnotic potency of sevoflurane in patients with sleep disorders differ from patients with normal sleep habits.

Methods: We recruited 44 patients scheduled for elective breast surgery and eventually analyzed 38 patients, including 19 subjects with normal sleep habits and 19 subjects with sleep disorders. According to the Dixon 'upand-down' design, patients received sevoflurane at preselected concentrations starting at 1.0 vol\%. After a steadystate period, a verbal command for testing awakening was performed. Based on the negative or positive response to the verbal command, we decreased or increased the concentration of sevoflurane by $0.2 \mathrm{vol} \%$ in the next patient accordingly. Plasma orexin-A was also measured before observation.

Results: The MACawake of sevoflurane was $0.80 \%$ [95\% confidence interval (CI), 0.683-0.926\%] in the sleep disordered group vs $0.60 \%$ [95\% Cl, 0.493-0.689\%] in the control group. The relative median potency between groups was 0.750 (95\% Cl, 0.236-0.969). Patients with sleep disorders had significantly higher orexin-A levels than control $(72.17 \pm 18.24$ vs. $36.16 \pm 14.18 \mathrm{pg} / \mathrm{mL})$. A significant, positive relationship was detected between orexin-A level and probability of awakening $(\mathrm{OR}=1.081,95 \% \mathrm{Cl}$ is $1.020-1.146, P=0.008)$.

Conclusions: MACawake of sevoflurane is higher in mild-aged women of breast surgery with sleep disorders compared to those with normal sleep habits. The increased anesthetic requirement may be related to changes of orexin-A levels. These findings suggest that sleep may have a potential impact on clinical anesthesia, including changes of sensitivity to anesthetics or postoperative complications. Further research is needed to confirm this hypothesis.
\end{abstract}

Clinical trial registration: Chinese Clinical Trial Registry (ChiCTR1800016022), date of registration 07 May 2018.

Keywords: Sevoflurane, MACawake (minimum alveolar concentration of awake), Orexin-A, Sleep disorders

\section{Background}

Sleep disorders such as insomnia are commonly encountered in clinic because of fear, anxiety, pain, or a disruptive environment. Patients with sleep disorders are more likely to experience postoperative complication like delirium $[1,2]$. It has been shown that sleep deprivation can modulate the effectiveness of general

\footnotetext{
* Correspondence: zhangye_hassan@sina.com

${ }^{1}$ Department of Anesthesiology, Second Affiliated Hospital of Anhui Medical University, 678 Furong Rd, Hefei, Anhui, China

Full list of author information is available at the end of the article
}

anesthetics in rats, including hypersensitivity to induction of anesthesia, or delayed emergence from anesthesia $[3,4]$. However, the mechanism of sleep changes affect general anesthesia remains unclear.

It has been speculated that common neural pathways between natural sleep and general anesthesia may regulate emergence from anesthesia [5]. Orexins, especially orexin-A, play a crucial role in the promotion and maintenance of wakefulness during anesthesia emergence [6, 7]. One previous study showed that a reduction of Orexin-A is responsible for prolonged emergence from

(c) The Author(s). 2020 Open Access This article is distributed under the terms of the Creative Commons Attribution 4.0 International License (http://creativecommons.org/licenses/by/4.0/), which permits unrestricted use, distribution, and 
isoflurane anesthesia in rats subjected to sleep deprivation [4]. However, this theory has yet to be confirmed in humans. Therefore, we hypothesized that sleep disorders along with a change of orexin-A might modulate the hypnotic potency of anesthetics in humans.

To test this, we assessed the minimum alveolar concentration (MAC) of inhaled anesthetic agents to achieve a $50 \%$ probability of nonresponsive to a stimulus (MACawake), which provides a measure of hypnotic potency. Considering that sevoflurane is the most commonly used volatile anesthetics for general anesthesia and sedation, the aim of our study was to determine whether the MACawake of sevoflurane in women with sleep disorders are different to those with normal sleep patterns (control). Also, we aimed to determine a correlation between the probability of awaking and plasma concentrations of orexin-A.

\section{Methods \\ Participants}

This study was approved by our local Institutional Ethical Committee (First Affiliated Hospital of Anhui Medical University, and informed consent was obtained preoperatively from each patient. We enrolled 44 women aged 40-60 yrs. undergoing elective breast surgery using general anesthesia from May 2018 to September 2018 at First Affiliated Hospital of Anhui Medical University, Hefei, China.

All participants were classified by the American Society of Anesthesiologists physical status classification of I or II, without dysaudia. Patients were excluded if they presented with any of these criteria: anemia, hypoxia, hypotension, hypercapnia, abnormal acid-base status, electrolyte disturbances, fever, or obesity (body mass index $>30 \mathrm{~kg} \mathrm{~m}^{2}$ ); associated with any neurological disease and psychotropic medications; daily alcohol consumption; history of drug dependence or use of over-the-counter sleep medications within the previous 6 months. All patients gave written, informed consent.

The following inclusion criteria were used for subjects with sleep disorders [8]: difficulty initiating or maintaining sleep $\geq 6$ months, with Pittsburgh Sleep Quality Index (PSQI) $\geq 7$, according to the Diagnostic and Statistical Manual of Mental Disorders (DSM-V) criteria [9]; Hamilton Anxiety Scale (HAMA) < 7; and Hamilton Depression Scale $(\mathrm{HAMD})<7$. The following criteria were used for normal sleepers [8]: no history or evidence of sleep disorders; PSQI < 7, HAMA and HAMD scores less than 7.

All subjects were interviewed and assessed for PSQI, HAMA, and HAMD by an trained anesthesiologist on the day before surgery. Written informed consent was obtained at the same time.

\section{Up and down method}

All patients had fasted for $8 \mathrm{~h}$ and received no sedative or anesthesia drugs prior to the observation. Standard monitoring was conducted throughout the observation, including electrocardiogram, noninvasive blood pressure, heart rate, pulse oximeter, and inhaled gas analysis (carbon dioxide and sevoflurane).

General anesthesia was induced by breathing $8 \%$ sevoflurane (Maruishi Pharmaceutical Co, Ltd., Osaka, Japan) mixed with $100 \%$ oxygen at a flow rate of $61 \mathrm{~min}^{-1}$ through a semi-closed face mask, delivered through a semi closed circuit system (Sevotec 7; Datex- Ohmeda Inc., Madison, WI, USA). The inspired and end tidal concentrations of sevoflurane and end tidal carbon dioxide in $\mathrm{mmHg}$ were sampled through a tube connected to the distal end of the mask and analyzed by a gas analyzer (Philips G60 Series Patient Monitor, Philips, Böblingen, Germany).

After loss of the lash reflex, the target concentration of the first patient was adjusted to $1.0 \%$ in $100 \%$ oxygen at a flow rate of $2 \mathrm{~L} \mathrm{~min}^{-1}$ in both two groups. After maintaining for $15 \mathrm{~min}$, verbal commands were given to test awakening by an independent observer who was blinded to the design and the aim of the study. The observer asked the patient to open their eyes with a normal tone, and repeated 3 times. The response of each patient was record as 'no response' or 'response'. According to the "up and down" method [10], the target concentration of next patient was dependent on the response of the previously tested patient. If the previous patient responded, the target concentration was set at $0.2 \%$ higher, but the target concentration was set at $0.2 \%$ lower if the previous patient did not respond to the command.

The operating room was kept quiet and at a temperature ranging from 24 to $26^{\circ} \mathrm{C}$. The warmed lactated Ringer's was injected at a rate of $8-10 \mathrm{~mL} \mathrm{~kg}^{-1}$ $\mathrm{h}^{-1}$. Ventilation was assisted if the end-tidal carbon dioxide $\left(\mathrm{ETCO}_{2}\right)$ level was $>45 \mathrm{mmHg}$, or if the tidal volume was too low $(<500 \mathrm{~mL})$. Phenylephrine $40 \mu \mathrm{g}$ was administered i.v. if necessary to maintain MAP. A previous observer blinded to the study recorded the data. After the observation, anesthesia was deepened appropriately for tracheal intubation and surgery.

\section{Measurement of orexin-a}

Blood samples $(5 \mathrm{~mL})$ were collected into EDTA tubes before observation for blood gas analysis and then transferred into centrifuge tubes containing aprotinin. The blood samples were centrifuged at $1600 \mathrm{rpm}$ for $15 \mathrm{~min}$ at $4{ }^{\circ} \mathrm{C}$ to separate plasma and then stored at $-80^{\circ} \mathrm{C}$ for assay later. Plasma orexin-A was measured by a Chemiluminescent EIA kit (Phoenix pharmaceuticals Inc., Burlingame, California, USA). 


\section{Statistical analysis}

All statistical analyses were performed using SPSS software version 19.0 (SPSS for MAC, Chicago, IL, USA). Patient characteristic data were presented as means (SD). Patient characteristics, blood glucose, liquid volume and $\mathrm{ETCO}_{2}$ were compared between groups by independent-samples t-test or nonparametric test.

$\mathrm{Up}$ and down sequences were analyzed by the probit test, which enabled estimation of MACawake with 95\% CI of the mean. The calculated MACawake of sevoflurane between groups was compared using the estimate of relative median potency, which is the ratio of sevoflurane concentration needed to obtain a $50 \%$ probability of being awake in each group. Logistic regression was conducted to test the correlation of probability of awakening and Orexin-A. $P$ values of $<0.05$ were considered statistically significant.

In our previous study, we determined a MACawake of $0.67 \%$ for patients 50 yrs. old [11]. We conducted an a priori power analysis to detect a difference of $0.2 \%$ (SD = 0.2 ) in the concentration of sevoflurane. On the assumption of power of 0.80 and a type I error rate of 0.05 , we needed to enroll 16 patients for each group.

\section{Results}

Forty-four patients completed the investigation as shown in Fig. 1. Six patients were excluded from the study. Five were excluded from the observation (2 did not give consent, 1 was obese, 1 had severe anemia, and 1 was using midazolam before observation) and one patient was excluded from the analysis for wracking cough during the observation. No hypotension (systolic blood pressure < $60 \mathrm{mmHg}$ ), bradycardia (heart rate $<50 \mathrm{bpm}$ ), oxygen desaturation $\left(\mathrm{SpO}_{2}<95 \%\right)$, or respiratory depression was observed during the study. There is no significant difference in patient characteristics, liquid volume, or $\mathrm{ETCO}_{2}$ between the two groups. Orexin-A levels were significantly higher in the sleep disordered group (Table 1).

Fig. 2 shows the individual responses to the vocal command according to the up and down sequence. The $50 \%$ effective dose for MACawake was $0.80 \%$ [95\% CI, $0.68-0.93 \%$ ] and $0.60 \%$ [95\% CI, $0.49-0.69 \%$ ] for the sleep disordered and control group, respectively, and the relative median potency was 0.749 (95\% CI, 0.24-0.97). There was a difference in the median potency of the 2 groups because the CI did not include one. Fig. 3 depicts the dose-response curve of the probability of awake between the two groups. Logistic regression analysis showed that the equation of the logistic regression analysis was Pawakening $=9.595+0.078$ Orexin-A, with OR of $1.081(95 \% \mathrm{CI}$ is $1.020-1.146, P=0.008)$ (Table 2).

\section{Discussion}

Our study demonstrated that the MACawake of sevoflurane in $100 \%$ oxygen in mild-aged women of breast surgery with sleep disorders was higher compared to those with normal sleep. Additionally, patients with sleep disorders exhibited significantly higher orexin-A

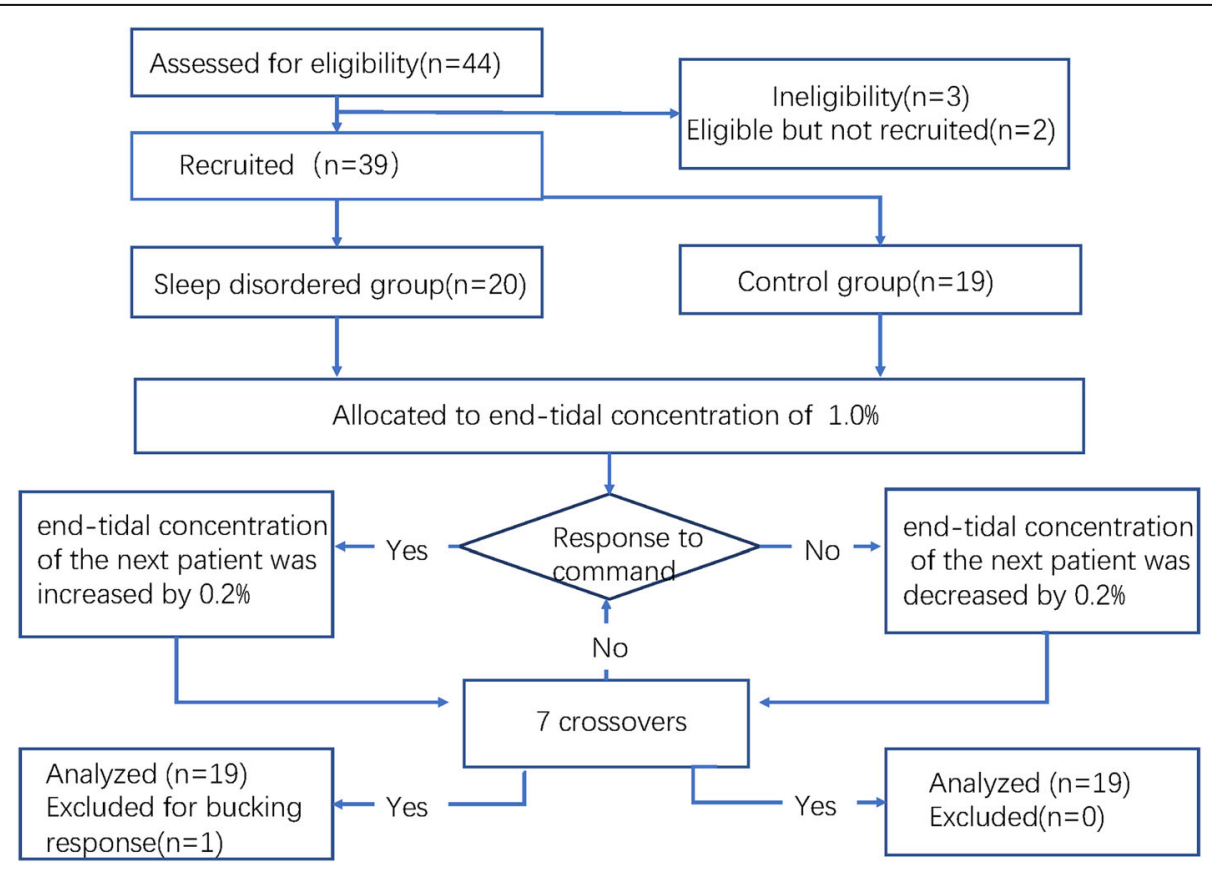

Fig. 1 Flow diagram for the Dixon up and down method. Five patients were excluded from observation (2 without consent and 1 with obesity and 1 with severe anemia and 1 for midazolam was used before observation); one patient was excluded from analysis for serious cough 
Table 1 Subject characteristics and concentration of orexin-A

\begin{tabular}{llll}
\hline Parameters & Sleep Disordered & Control & $P$ \\
\hline Age (yrs) & $50.8 \pm 2.9$ & $49.9 \pm 4.7$ & 0.708 \\
Body weight $(\mathrm{kg})$ & $63.4 \pm 6.9$ & $59.8 \pm 7.2$ & 0.131 \\
Height $(\mathrm{cm})$ & $164 \pm 3$ & $161 \pm 4$ & 0.055 \\
Glucose $(\mathrm{mmol} / \mathrm{L})$ & $4.78 \pm 0.58$ & $4.96 \pm 0.61$ & 0.332 \\
ETCO $_{2}(\mathrm{mmHg})$ & $25.4 \pm 3.3$ & $26.4 \pm 3.2$ & 0.327 \\
Liquid volume $(\mathrm{mL})^{405 \pm 58}$ & $393 \pm 61$ & 0.536 \\
Orexin-A $(\mathrm{pg} / \mathrm{mL})$ & $72.2 \pm 18.2$ & $36.2 \pm 14.2$ & $<0.001^{*}$
\end{tabular}

Data are presented as means $\pm \mathrm{SD}$. $\mathrm{ETCO}_{2}$ was recorded before verbal commands. Liquid volume data collection started from the time entering the room until the end of observation. ${ }^{*} P<0.05$ versus control

levels than normal sleepers. Moreover, a positive correlation between probability of awakening and plasma orexin-A concertation was confirmed.

The Dixon "up and down" method is an effective statistical approach for calculation of MAC [ 12, 13]. Six pairs are optimal for a clinical study, and the reliability increases with an increasing number of pairs [14]. Based on the calculated sample size, we stopped the observation after seven pairs of opposite responses in the two groups. We derived the MACawake of patients with

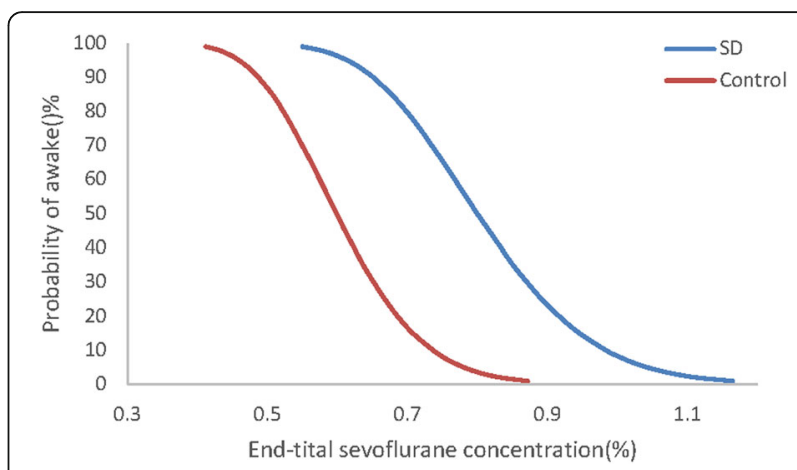

Fig. 3 The dose-response curve from the probit analysis of end-tidal sevoflurane concentrations and probability of being awake. Minimum alveolar concentration-awake (MACawake) in patients with sleep disorders was $0.8 \%$ [95\% Cl, 0.683-0.926\%]; MACawake in the control group was $0.60 \%$ [95\% Cl, 0.493-0.689\%]

normal sleep to be $0.60 \%$, consistent with previous work [15] which demonstrated a MACawake of $0.63 \%$.

As far as we know, there are no quantified studies of MACawake of sevoflurane in humans with sleep disorders. The mechanism of increased awakening concentration of sevoflurane is unclear. There are many factors which can affect the potency of inhaled anesthetics [16],
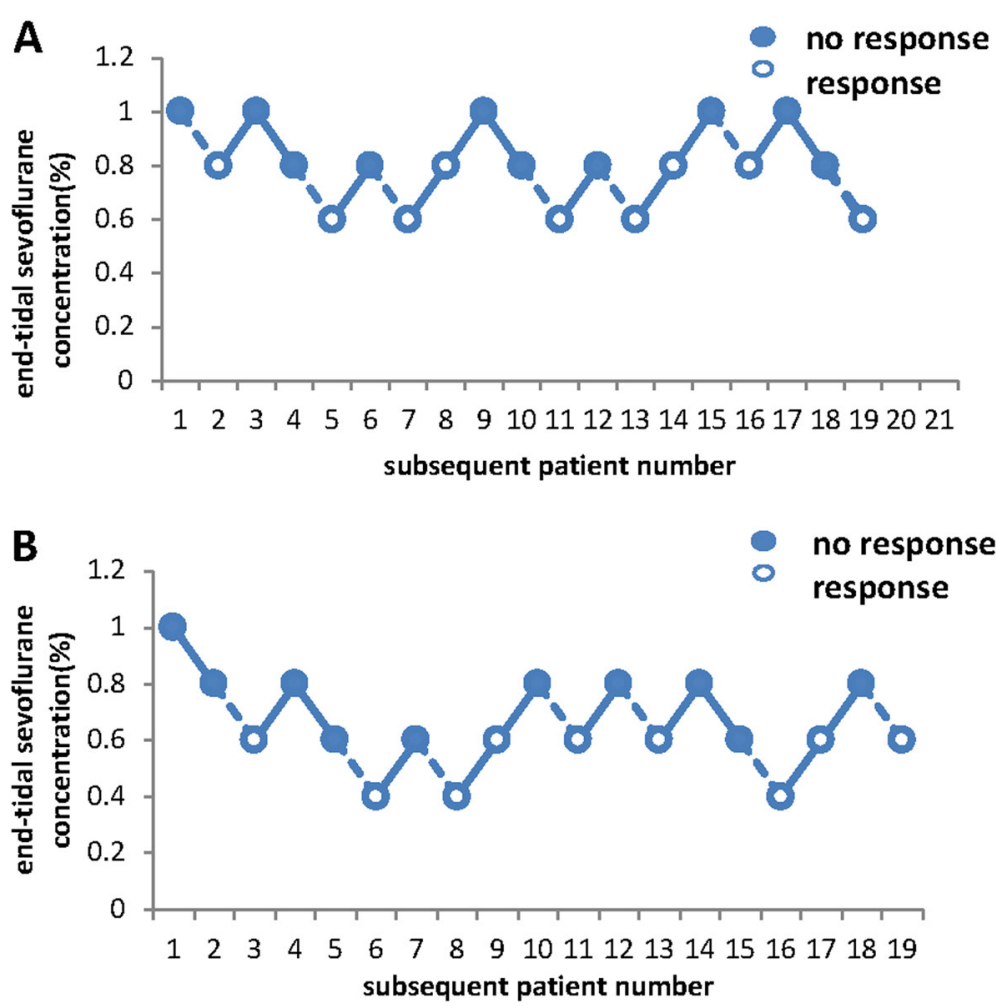

Fig. 2 Response of each subject to predetermined end-tidal sevofluane concentrations. Closes circles represent no response to the vocal command and open circles represent response to command. The dotted line between points of and showed seven crossovers of responses. a, MACawake of sevoflurane in patients with sleep disorders. b, MACawake of sevoflurane in the control group 
Table 2 Results of logistic regression analysis for $P_{\text {awakening }}$ as the dependent variable

\begin{tabular}{llllll}
\hline Independent variable & Regression coefficient $(\beta)$ & SE & OR & $95 \%$ Cl for OR & $P$ value \\
\hline Constant & 9.595 & 3.684 & 14,684 & - & 0.009 \\
Orexin-A & 0.078 & 0.030 & 1.081 & $1.020-1.146$ & 0.008 \\
Concentration of sevoflurane (\%) & -19.937 & 6.609 & 0.000 & $0.000-0.001$ & 0.003 \\
\hline
\end{tabular}

$\mathrm{Cl}$ confidence interval, SE standard error

such as age or pathophysiological conditions. However, these have been ruled out in the current study. Finally, we conducted the observation only during anesthesia induction to minimize any surgical confounders. Therefore, the change of hypnotic potency was likely attributed to changes in the functional status of the brain as a consequence of sleep disorders.

The orexin system regulates the sleep-wake cycle [17]. Narcolepsy is caused by impaired orexinergic signaling, as seen by lower levels of orexin-A [18]. Genetic and pharmacological blockades of orexin-mediated signaling impact arousal [6]. Conversely, patients with insomnia disorders exhibit higher orexin-A levels, which is consistent with our study [8]. Previous work has also demonstrated a significant correlation between plasma orexin-A and arousal index or Epworth sleepiness scale score $[5,19]$. This relationship highlights the critical role of orexin in maintaining wakefulness. Orexinergic neurons may therefore also affect general anesthesia. Animal studies suggest that orexin signaling can modify the anesthetic state, as activation of the orexinergic signals can significantly promote emergence from inhalation [20] or intravenous [21] anesthesia. Additionally, inhibition of orexinergic signaling prolongs awakening time from anesthesia state, impairs arousal, and increases anesthetic duration $[5,22,23]$. Therefore, we propose that an increase in orexin-A may increase the MACawake in patients with sleep disorders.

This study has several limitations which must be addressed. First, all subjects were middle-aged women, which might lead to Berkson's bias without an elderly control comparison. Also, elderly patients who are more likely to have sleep disorders and thus have serious complications of general anesthesia should be studied in future investigations. Second, we did not measure orexinA in the cerebrospinal fluid for ethical reasons. Plasma orexin-A originates from the central nervous system (CNS), the pancreas [24], and the gut [25]. However, release of orexin-A from the hypothalamus would be much greater compared to peripheral tissues [26]. Additionally, orexin-A can rapidly cross the blood-brain barrier [27], and thus we were required to assume that plasma levels of orexin-A reflect the CNS release of orexin-A. At last, sleep disorders are distinguished by the PSQI scale, which provide a comprehensive assessment. Thus, the causes and types of sleep disorders were not classified, and we did not distinguish between sleep conditions at home and after admission, but those need to be study in the further research.

\section{Conclusion}

We demonstrate that the MACawake of sevoflurane were significantly increased in mild-aged women of breast surgery with sleep disorders compared to women with normal sleep patterns. The potential mechanism may be related to change of orexin-A levels. Further studies are needed to confirm our results. More researches need to explore that if sleep disorder may change efficacy of other general anesthetics, and whether decrease in hypnotic potency of general anesthetics are associated with postoperative delirium.

\section{Supplementary information}

Supplementary information accompanies this paper at https://doi.org/10. 1186/s12871-020-0931-3.

Additional file 1. Experimental data.

\section{Abbreviations}

Cl: Confidence interval; CNS: Central nervous system; DSM-V: Diagnostic and Statistical Manual of Mental Disorders; $\mathrm{ETCO}_{2}$ : End-tidal carbon dioxide; HAMA: Hamilton Anxiety Scale; HAMD: Hamilton Depression Scale; MAC: Minimum alveolar concentration; MACawake: MAC of inhaled anesthetic agents to achieve a 50\% probability of being awake;

PSQI: Pittsburgh Sleep Quality Index; SE: Standard error

\section{Acknowledgments}

We thank LetPub (www.letpub.com) for its linguistic assistance during the preparation of this manuscript. Also, we like to thank the participating patients for their contribution to this study.

\section{Authors' contributions}

Study design: YYC,YW,YZ. Study conduct: YYC, XHP,LZ. Data analysis: YW,QLZ. Writing paper: YYC, EWG, YZ. All authors read and approved the final manuscript.

\section{Funding}

This research received no specific grant from any funding agency in the public.

Availability of data and materials

The datasets supporting the conclusions of this article is included within the article (and its Additional file 1).

\section{Ethics approval and consent to participate}

The study was approved by the ethics committee of First Affiliated Hospital of Anhui Medical University on 29 March 2018(approved number:PJ2018-0310). Written informed consents have been obtained from all patients. 


\section{Consent for publication}

Not applicable.

\section{Competing interests}

The authors declare that they have no competing interests.

\section{Author details}

'Department of Anesthesiology, Second Affiliated Hospital of Anhui Medical University, 678 Furong Rd, Hefei, Anhui, China. ${ }^{2}$ Department of Anesthesiology, First Affiliated Hospital of Anhui Medical University, 218 Jixi Rd, Hefei, Anhui, China. ${ }^{3}$ Department of Pharmaceutical Analysis, Anhui Medical University, Hefei, Anhui, China.

Received: 17 January 2019 Accepted: 7 January 2020 1. 010

\section{References}

1. Todd OM, Gelrich L, MacLullich AM, et al. Sleep disruption at home as an independent risk factor for postoperative delirium. J Am Geriatr Soc. 2017; 65(5):949-57.

2. Leung JM, Sands LP, Newman S, et al. Preoperative sleep disruption and postoperative delirium. J Clin Sleep Med. 2015;11(8):907-13.

3. Tung A, Szafran MJ, Bluhm B, et al. Sleep deprivation potentiates the onset and duration of loss of righting reflex induced by propofol and isoflurane. Anesthesiol. 2002;97(4):906-11.

4. Ran MZ, Wu W, Li JN, et al. Reduction of orexin-a is responsible for prolonged emergence of the rat subjected to sleep deprivation from isoflurane anesthesia. CNS Neurosci Ther. 2015;21(3):298-300.

5. Franks NP. General anaesthesia: from molecular targets to neuronal pathways of sleep and arousal. Nat Rev Neurosci. 2008:9(5):370-86.

6. Chemelli RM, Willie JT, Sinton CM, et al. Narcolepsy in orexin knockout mice: molecular genetics of sleep regulation. Cell. 1999;98(4):437-51.

7. Kushikata T, Hirota $\mathrm{K}$, Yoshida $\mathrm{H}$, et al. Orexinergic neurons and barbiturate anesthesia. Neurosci. 2003;121(4):855-63.

8. Tang S, Huang W, Lu S, et al. Increased plasma orexin-a levels in patients with insomnia disorder are not associated with prepro-orexin or orexin receptor gene polymorphisms. Pept. 2017:88:55-61.

9. Reynolds CF 3rd, O'Hara R. DSM-5 sleep-wake disorders classification: overview for use in clinical practice. Am J Psychiatry. 2013;170(10):1099-101.

10. Zou ZY, Zhao YL, Yang XL, et al. Effects of different remifentanil target concentrations on MAC BAR of sevoflurane in gynaecological patients with CO2 pneumoperitoneum stimulus. Br J Anaesth. 2015;114(4):634-9.

11. Wu Y, Jin $S$, Zhang L, et al. Minimum alveolar concentration-awake of Sevoflurane is decreased in patients with end-stage renal disease. Anesth Analg. 2018;128(1):77-82

12. Bourgeois E, Sabourdin N, Louvet N, et al. Minimal alveolar concentration of sevoflurane inhibiting the reflex pupillary dilatation after noxious stimulation in children and young adults. Br J Anaesth. 2012;108(4):648-54.

13. Niu B, Fang Y, Miao JM, et al. Minimal alveolar concentration of sevoflurane for induction of isoelectric electroencephalogram in middle-aged adults. $\mathrm{Br}$ J Anaesth. 2014;112(1):72-8.

14. Paul M, Fisher DM. Are estimates of MAC reliable? Anesthesiol. 2001;95(6): 1362-70.

15. Katoh T, Ikeda K, Bito H. Does nitrous oxide antagonize sevoflurane-induced hypnosis? Br J Anaesth. 1997;79(4):465-8.

16. Quasha AL, Eger EI 2nd, Tinker JH. Determination and applications of MAC Anesthesiol. 1980;53(4):315-34.

17. Hirota K, Kushikata T. Central noradrenergic neurones and the mechanism of general anaesthesia. Br J Anaesth. 2001:87(6):811-3.

18. Mignot E, Lammers GJ, Ripley B, et al. The role of cerebrospinal fluid hypocretin measurement in the diagnosis of narcolepsy and other hypersomnias. Arch Neurol. 2002;59(10):1553-62.

19. Sakurai S, Nishijima T, Takahashi S, et al. Low plasma orexin-a levels were improved by continuous positive airway pressure treatment in patients with severe obstructive sleep apnea-hypopnea syndrome. Chest. 2005;127(3):731-7.

20. Dong H, Niu J, Su B, et al. Activation of orexin signal in basal forebrain facilitates the emergence from sevoflurane anesthesia in rat. Neuropeptides. 2009:43(3):179-85.

21. Zhang LN, Li ZJ, Tong L, et al. Orexin-a facilitates emergence from propofol anesthesia in the rat. Anesth Analg. 2012;115(4):789-96.
22. Kelz MB, Sun Y, Chen J, et al. An essential role for orexins in emergence from general anesthesia. Proc Natl Acad Sci U S A. 2008;105(4):1309-14.

23. Yasuda Y, Takeda A, Fukuda S, et al. Orexin a elicits arousal electroencephalography without sympathetic cardiovascular activation in isoflurane-anesthetized rats. Anesth Analg. 2003;97(6):1663-6.

24. Ouedraogo R, Naslund E, Kirchgessner AL. Glucose regulates the release of orexin-a from the endocrine pancreas. Diabetes. 2003;52(1):111-7.

25. Kirchgessner AL. Orexins in the brain-gut axis. Endocr Rev. 2002;23(1):1-15.

26. Taheri S, Mahmoodi M, Opacka-Juffry J, et al. Distribution and quantification of immunoreactive orexin a in rat tissues. FEBS Lett. 1999;457(1):157-61.

27. Kastin AJ, Akerstrom V. Orexin a but not orexin B rapidly enters brain from blood by simple diffusion. J Pharmacol Exp Ther. 1999;289(1):219-23.

\section{Publisher's Note}

Springer Nature remains neutral with regard to jurisdictional claims in published maps and institutional affiliations.
Ready to submit your research? Choose BMC and benefit from:

- fast, convenient online submission

- thorough peer review by experienced researchers in your field

- rapid publication on acceptance

- support for research data, including large and complex data types

- gold Open Access which fosters wider collaboration and increased citations

- maximum visibility for your research: over $100 \mathrm{M}$ website views per year

At BMC, research is always in progress.

Learn more biomedcentral.com/submissions 